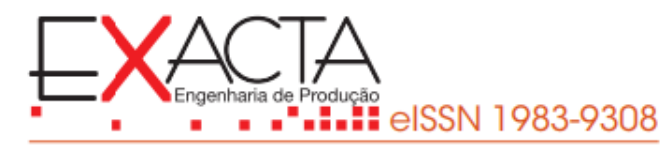

\title{
MODELO DE FLUXO DE REDE PARA SELEÇÃO DE FORNECEDORES EM RESPOSTA AOS DESASTRES: UM EXERCÍCIO NA REGIÃO SERRANA DO ESTADO DE SANTA CATARINA - BRASIL
}

\section{NETWORK FLOW MODEL FOR THE SELECTION OF SUPPLIERS IN RESPONSE TO DISASTERS: AN EXERCISE IN THE MOUNTAIN REGION AT THE STATE OF SANTA CATARINA, BRAZIL}

Recebido em: 18 maio. 2020

Aprovado em: 11 ago. 2020

Versão do autor aceita publicada online: 11 ago. 2020

Publicado online: 25 jun. 2021

Como citar esse artigo - American Psychological Association (APA):

Lima, F. S., Oliveira, D., Trierweiller, A. C., \& Vefago, Y. (2022, jan./mar.). Modelo de fluxo de rede para seleção de fornecedores em resposta aos desastres: um exercício na região serrana do estado de Santa Catarina - Brasil. Exacta. 20(1), 198-217. https://doi.org/10.5585/exactaep.2021.17236.

Submeta seu artigo para este periódico $\bigoplus$

Dados Crossmark 


\title{
MODELO DE FLUXO DE REDE PARA SELEÇÃO DE FORNECEDORES EM RESPOSTA AOS DESASTRES: UM EXERCÍCIO NA REGIÃO SERRANA DO ESTADO DE SANTA CATARINA - BRASIL
}

\author{
NETWORK FLOW MODEL FOR THE SELECTION OF SUPPLIERS IN RESPONSE TO \\ DISASTERS: AN EXERCISE IN THE MOUNTAIN REGION AT THE STATE OF SANTA \\ CATARINA, BRAZIL
}

\author{
iD Fabiana Santos Lima ${ }^{1}$ \\ iD Daniel de Oliveira ${ }^{2}$ \\ iD Andrea Cristina Trierweiller ${ }^{3}$ \\ iD Yuri Vefago 4
}

${ }^{1}$ Doutora

Universidade Federal de Santa Catarina - UFSC.

Florianópolis, Santa Catarina - Brasil.

fabiana.lima@ufsc.com

2 Doutor

Universidade do Grande Rio José de Souza Herdy UNIGRANRIO

Duque de Caxias, Rio de Janeiro - Brasil.

daniel.oliveira@unigranrio.edu.br

${ }^{3}$ Doutora

Universidade Federal de Santa Catarina - UFSC. Araranguá, Santa Catarina - Brasil.

andrea.ct@ufsc.br

${ }^{4}$ Mestre

Universidade Federal de Santa Catarina - UFSC. Araranguá, Santa Catarina - Brasil. yuri.vefago@posgrad.ufsc.br

Recebido em: 18 maio 2020

Aprovado em: 11 ago. 2020
Resumo: Desastres afetam a normalidade da região atingida, causam danos e perdas em todo o sistema econômico social da região. Operações de logística humanitária buscam atender o mais rápido possível a demanda requerida, tendo como principal objetivo salvar o maior número de pessoas. Apresenta-se um modelo matemático e computacional para apoiar as operações humanitárias em eventos de desastres, com destaque para a função de aquisição de suprimentos. Fornecedores foram selecionados com a possibilidade de entregar os itens de assistência humanitária em curto prazo, utilizando um modelo de fluxo em redes adaptado, utilizou-se técnicas de simulação e otimização. Aplicou-se o modelo proposto no cenário de um evento adverso ocorrido no estado de Santa Catarina. Como resultado do problema de fluxo em redes, verificou-se que a condição do produto estar o mais rápido possível no local de demanda foi satisfeita e foi utilizada a capacidade dos fornecedores nas datas mais próximas aos eventos.

Palavras-chave: Logística humanitária. Fluxo em redes. Lead time. Desastres.

Abstract: Disasters affect the normality of affected region, causing damage and loss throughout the region's social and economic system. Humanitarian logistics operations seek to attend as soon as possible the required demand, with the main objective of saving the largest number of people. The aim of this study is to present a mathematical and computational model to support humanitarian operations in disaster events with emphasis on procurement function of supplies. Suppliers were selected with the possibility to deliver the items in short time, using networks flow model adapted, we used techniques of simulation and optimization. It was applied to the proposed model in scenario of adverse event occurred in the state of Santa Catarina. The result of the network flow problem, it was found that the product condition be as fast as possible on demand site was satisfied and we used the capacity of the suppliers in the most nearby dates.

Keywords: Humanitarian logistics. Network flow. Lead time. Disasters. 


\section{Introdução}

No contexto geral, o desastre pode ser definido como uma forte interrupção no funcionamento de uma nação. Segundo a Classificação e Codificação Brasileira de Desastres (Cobrade, 2020), os desastres podem ser de origem humana (causados pelo homem), como guerra e terrorismo; e de origem natural ou tecnológica. Por exemplo, o rompimento de uma barragem, como a que ocorreu em Brumandinho (MG) em janeiro de 2019, é considerado um incidente tecnológico pertencente ao grupo referente a desastres relacionados a obras civis. Por outro lado, os terremotos ocorridos em janeiro de 2010 no Haiti, são considerados desastres naturais do grupo geológico. Mais recentemente, tem-se o COVID-19, vírus que atualmente está atingindo o mundo inteiro, que também é considerado desastre natural, pertencente ao grupo biológico.

Cada grupo de desastres está relacionado a um subgrupo, cada subgrupo apresenta o conceito e sua definição, o que auxilia no tipo de ação a ser trabalhada no atendimento ao desastre. Algumas vezes, desastres são imprevisíveis e, dependendo da sua intensidade, podem devastar uma pequena região, assim como todo um país. O desastre, independente da origem, é uma situação de anormalidade que envolve perdas significativas nos mais diversos setores: econômico, humano e ambiental (ONU, 2009).

A volta ao estado de normalidade está diretamente associada à escala de devastação, a qual é influenciada pelo impacto do desastre, podendo ser de início súbito e com grande potencial de destruição, como é o caso dos terremotos, tsunami e eventos como de início lento, como ocorre com as secas.

O atendimento à região atingida requer planejamento, coordenação e a execução de ações de resposta e de recuperação aos desastres, desenvolvendo ações prioritárias para capacidade de resposta individual e coletiva, buscando a proteção da vida, do meio ambiente e do patrimônio (Lima, Eyerkaufer \& Gonçalves, 2017).

$\mathrm{Na}$ abordagem referente às ações prioritárias, segundo o Marco de Sendai (2015), o investimento público e privado, através de medidas estruturais e não estruturais, é essencial para melhorar a resiliência econômica, social, cultural e de saúde de pessoas, comunidades, países e ainda, do meio ambiente. Desta forma, como medidas estruturais, considera-se a participação de organizações de ordem pública, controladas pelo Estado (Secretaria da Defesa Civil - SDC, Corpo de Bombeiros e Exército); instituições privadas (empresas e fornecedores), onde o capital está nas mãos de particulares; e ainda, organizações mistas (aeroportos e bancos), onde o capital é partilhado por particulares e pelo Estado. A logística pode ser uma das partes mais caras de resposta aos desastres. Alguns autores, 
estimam que até $80 \%$ dos recursos gastos em uma resposta a desastres é referente à logística (Thomas, 2004; Trunick, 2005; Van Wassenhove, 2006).

Atividades voltadas à recuperação de desastres envolvem, anualmente, bilhões de dólares, os quais devem ser direcionados ao atendimento humanitário para a redução da pobreza e promoção dos direitos humanos. O sistema econômico da região atingida pode ser muito comprometido e a ausência de uma boa gestão em desastres ocasiona uma grande perturbação política e social, refletindo no relacionamento com outros países (Rodriguez, Vitoriano \& Montero, 2012; Tatham \& Pettit, 2010; Olsen, Carstensen \& Hoyen, 2003).

O desempenho das atividades de prevenção, mitigação, preparação, resposta e recuperação, está relacionado com a integração do processo de gestão do risco e desastres. Este processo compreende a fase pré-desastres, considerada no período de normalidade, caracterizada pelos processos de (1) prevenção, (2) mitigação e (3) preparação.

O período de anormalidade, ocorre na fase de resposta aos desastres, caracterizada pelas ações de assistência e socorro. Já a recuperação, que também ocorre no período de anormalidade, se dá na fase pós-desastres e consiste nas ações de reabilitação e reconstrução (Lima, Eyerkaufer \& Gonçalves, 2017).

Para qualquer uma dessas três fases, a Logística Humanitária (LH) engloba o planejamento e um conjunto de ações, que são necessários para salvar vidas, deslocar pessoas e materiais, promover o fluxo de informação e gerenciar a aquisição, armazenagem, o transporte e a distribuição de suprimentos (Samed \& Gonçalves, 2017). Atividades humanitárias como compras, por exemplo, são classificadas como aquisição para a ajuda humanitária, em que a ênfase principal é sobre a velocidade e disponibilidade, a fim de salvar vidas e aliviar o sofrimento.

Embora alguns produtos adquiridos para ajuda em operações humanitárias sejam relativamente simples, outros podem ser bastante onerosos; mas independentemente do valor dos produtos, a principal preocupação é a entrega rápida itens de assistência humanitária (Taupiac, 2001). É importante ter sistemas e processos nos locais para facilitar a colaboração entre os atores, buscando a manutenção de altos níveis de eficiência no momento da resposta.

O principal objetivo do processo de aquisição é assegurar que a organização responsável pelas operações humanitárias disponha dos recursos materiais necessários para o atendimento às necessidades operacionais e de apoio. Neste processo, são realizadas as atividades de definição dos itens de assistência humanitária (Lima, Oliveira \& Gonçalves, 2014), pedido de produtos, seleção dos fornecedores, contratos de compra e avaliação de ofertas de fornecimento (Blecken, 2010). 
López-Vargas e Cárdenas-Aguirre (2018) realizaram uma revisão de literatura para identificar os fatores que facilitam e dificultam a coordenação da LH. Os autores estabeleceram que essa coordenação é condicionada pelo ambiente complexo inerente aos desastres, pelas capacidades organizacionais, bem como pela confiança e transparência que existem entre eles. Segundo os autores, essas descobertas visam promover o interesse em coordenação interorganizacional e melhoria dos processos de preparação e resposta a desastres.

Considerando que diferentes atores atuam no ambiente humanitário, eles identificaram que os processos estratégicos e operacionais devem ser realizados sob uma perspectiva de gerenciamento da cadeia de suprimentos, o que permitirá estabelecer processos mais claros de compra, armazenamento e distribuição, bem como a definição de papéis específicos de acordo com as capacidades organizacionais de cada ator.

Abordagens quantitativas têm sido propostas na bibliografia sobre a aquisição em operações de ajuda humanitária nos últimos anos. Destaca-se o trabalho desenvolvido por Trestrail, Paul e Maloni (2009), que descreveram uma ferramenta de apoio à decisão MIP (Mixed-Integer Programing), a fim de melhorar o planejamento de transporte marítimo e a estratégia de preços de licitação dos fornecedores de alimentos. Além disso, tem-se o estudo de Falasca e Zobel (2011), que desenvolveram modelos de decisão de compras para a ajuda humanitária. Ambos os trabalhos visaram situações específicas e não consideraram o tempo de atendimento na entrega de suprimentos durante a fase de resposta aos desastres naturais.

Assim, o objetivo deste artigo contribui com esta lacuna, ao definir o melhor conjunto de fornecedores de forma a respeitar o tempo, a demanda e a capacidade de entrega no lugar atingido. Para tanto, desenvolveu-se um modelo adaptado do problema de fluxo em redes.

Neste sentido este artigo apresenta, dentre suas contribuições: Levantamento dos atributos e aspectos relevantes na LH que devem ser contemplados para a elaboração do modelo de fluxo em redes; Desenvolvimento de técnicas de simulação e otimização, para estudar o impacto da aplicação do modelo deste modelo na LH; Implementação do método simplex especializado para resolver o problema de fluxo em redes adaptado à LH, utilizando a linguagem de programação Java; Utilização do simulador Anylogic ${ }^{\circledR}$ para a visualização na linha do tempo, no cenário analisado; Apresentação do índice de tempo para medir o desempenho no cenário. Afinal, na Logística Humanitária, devido ao custo de privação, o programa atende no tempo mais próximo da data de demanda, utilizando para isto, o fornecedor com capacidade suficiente para atender na data solicitada ou o mais próximo possível desta data. Quanto menor o tempo para atender totalmente a demanda melhor o desempenho do sistema. 
Assim, as abordagens de planejamento apresentadas podem ser configuradas na fase de preparação com antecedência a possíveis desastres, permitindo que as organizações se adaptem a diferentes cenários de crise para atuar nas fases de preparação e resposta.

A seção 1 abordou a Introdução, apresentando a motivação para o estudo, que prioriza o tempo de atendimento nas operações de LH, fundamental no planejamento de resposta aos desastres, também são apresentados o objetivo e as contribuições do artigo. A seção 2, de Fundamentação Teórica, apresenta definições, características e tipologias da: Logística Humanitária; Gestão de Desastres e Aquisição. A seção 3 apresenta a metodologia proposta, com as considerações iniciais e a modelagem do problema. A seção 4 aborda a apresentação do cenário estudado. Nas seções 5 e 6 , respectivamente, estão a Análise dos resultados obtidos e as Considerações finais, encerrando-se com as Referências.

\section{Fundamentação teórica}

\subsection{Logística humanitária}

A logística é o processo de planejar, implementar e controlar de maneira eficiente e eficaz: o fluxo e a armazenagem de bens, serviços e informação relacionada, desde o ponto de origem até o ponto de consumo com o propósito de adequar às necessidades dos clientes. Considera-se que Logística Humanitária (LH) é a função que é exigida para assegurar com eficiência e eficácia o fluxo de suprimentos, pessoas e informação com o propósito de salvar vidas e aliviar o sofrimento de pessoas vulneráveis (Thomas, 2004). São processos e sistemas envolvidos na mobilização de pessoas, recursos e conhecimentos para ajudar comunidades vulneráveis afetadas por desastres naturais ou emergências complexas.

LH busca a pronta resposta visando atender o maior número de pessoas, evitar falta e desperdício, organizar as diversas doações que são recebidas nestes casos e, principalmente atuar dentro de um orçamento limitado.

\subsection{Gestão de desastres}

Gestão de desastres é o conjunto de atividades destinadas a manter o controle sobre as situações de desastre e emergência e ainda, fornecer uma estrutura para ajudar pessoas em situação de risco, para evitar ou recuperar os efeitos do desastre. Trata de situações que ocorrem antes, durante e depois do desastre. 
Dentre os objetivos da gestão de desastres, tem-se: evitar ou reduzir as perdas humanas, físicas e econômicas sofridas pelas pessoas, pela sociedade e pelo país em geral; reduzir o sofrimento das pessoas; acelerar a recuperação da área afetada (Schultz, 2008).

É vista como um ciclo composto por quatro fases:

a) Prevenção de desastres: conjunto de ações destinadas a reduzir a ocorrência e a intensidade de desastres.

b) Preparação para emergências e desastres: conjunto de ações desenvolvidas pela comunidade/instituições governamentais e não governamentais, para minimizar os efeitos dos desastres.

c) Resposta aos desastres: conjunto de ações desenvolvidas imediatamente após a ocorrência de um desastre e caracterizadas por atividades de socorro, atividades de assistência às populações afetadas, atividades de reabilitação de cenários.

d) Reconstrução: conjunto de ações destinadas a recuperar a infraestrutura e a restabelecer, em sua plenitude, os serviços públicos, a economia da área e o bem-estar da população.

As fases de preparação e resposta à emergência, focos deste estudo, implicam em estabelecer processos, mecanismos e materiais que podem ser confiáveis para responder ao ambiente dinâmico de desastres e apoiar uma resposta ágil, adaptável e alinhada.

Algumas decisões logísticas na fase de preparação consistem em definir se suprimentos devem ser pré-posicionados e, em quais locais deve ocorrer esse pré-posicionamento, de forma a possibilite um fluxo ágil ao longo da cadeia de assistência humanitária. Imediatamente após a ocorrência do desastre, as decisões logísticas de resposta consistem em fazer com que os suprimentos cheguem aonde são necessários (Samed \& Gonçalves, 2017)

O início de um desastre é um grande desafio. A resposta de demanda imediata necessita da mercadoria certa distribuída na hora e local certos, bem como para as pessoas certas. Várias são as atividades voltadas para este atendimento em organizações humanitárias que auxiliam no processo de decisão como, por exemplo, armazenagem, transporte e aquisição.

A análise das atividades propostas à aplicação deste modelo refere-se às tarefas relacionadas com a aquisição a qual é descrita com mais detalhes e pode ser adaptada e estendida para ser aplicada, utilizando-se ferramentas quantitativas para apoio à decisão em LH. 


\subsection{Aquisição}

Segundo Schulz e Heigh (2009), 65\% do orçamento total de alívio de desastre é destinado à aquisição de suprimentos e equipamentos de socorro. A complexidade das operações de aquisição deve-se a alguns fatores como, por exemplo, os mecanismos de financiamento, as esperas e expectativas dos doadores, a diversidade das partes interessadas, a imprevisibilidade dos desastres, a escassez de recursos e o excesso de oferta (Balcik et al., 2010). Essa complexidade apresenta árduas questões às organizações humanitárias referentes à "que tipo, quanto, quando, onde e como" itens de assistência humanitária podem ser adquiridos.

No que diz respeito às operações de socorro, os métodos de aquisição em LH podem ser classificados quanto à localização dos fornecedores e quanto ao tempo de aquisição. Tanto localmente como globalmente, a aquisição antes do início do desastre aborda as fases de mitigação e preparação, enquanto, a aquisição após o início do desastre aborda as fases de resposta e recuperação. Os procedimentos operacionais como, por exemplo, tipos de contrato, disponibilidade de recursos, qualidade e preço dos produtos mudam de acordo com a localização dos fornecedores. Antes do início de desastres, as organizações humanitárias têm tempo suficiente para pesquisar vários fornecedores, comparar a sua qualidade e disponibilidade (Ertem \& Buyurgan, 2013).

As tarefas da aquisição incluem a identificação e a seleção de fornecedores adequados de forma a garantir a disponibilidade do produto nas áreas afetadas, no momento certo. São necessárias que sejam atendidas as especificações mínimas que satisfazem os critérios para condições específicas de operação e com o melhor preço disponível, tendo em conta o custo, prazo de entrega e qualidade, bem como o transporte razoável, seguro de transporte e os custos ligados à importação (Blecken, 2010).

O processo de aquisição de fornecedores locais é pouco aplicado antes do início do desastre, pois como a localidade do desastre é desconhecida não há como diferenciar fornecedores locais e fornecedores globais. Uma forma de incentivar a economia local seria a localização de um armazém global, desta forma, o contrato de fornecedores locais perto do armazém pode ser considerado, o que pode ser útil para o desenvolvimento econômico da região (Ertem \& Buyurgan, 2013). Os baixos custos de transporte e a entrega rápida podem ser outras vantagens do uso de fornecedores locais. Por outro lado, a qualidade pode não ser a esperada ou a capacidade dos fornecedores locais pode não ser suficiente para alta demanda de volume (Balcik \& Beamon 2008). O objetivo da aquisição é assegurar que a organização humanitária tenha os recursos materiais necessários para atender aos requisitos operacionais e os requisitos de suporte operacional (Blecken, 2010). 


\section{Metodologia proposta}

Utilizou-se pesquisa quantitativa, em que, de acordo com Cauchick (2010), nesta linha de pesquisa, a preocupação é assegurar que exista adesão entre observações e ações na realidade e o modelo elaborado da realidade. Conforme Coughlan e Coghlan (2002), a pesquisa empírica busca criar modelos que se encaixam bem com as relações causais existentes dentro do verdadeiro problema.

Para compreender como o método pode facilitar o apoio à decisão para diferentes indivíduos, bem como para tirar conclusões gerais e discutir as limitações e oportunidades de apoio à decisão durante os eventos de emergência foi usada simulação discreta, onde, alinha-se o funcionamento do sistema nos processos de aquisição no nível operacional da gestão ao longo de uma cadeia de fornecimento humanitário. Foi definida uma metodologia para organizar as atividades com o objetivo principal de reduzir o tempo de tomada de decisão nas operações humanitárias.

\subsection{Considerações iniciais}

Na modelagem, considerou-se a existência de parceria entre empresas privadas (fornecedores) e organizações humanitárias (Defesa Civil) em que, dados de identificação, localização, capacidade de fornecimento e preço são constantemente atualizados; considerou-se também que a responsabilidade do transporte dos produtos é do fornecedor, uso do sistema CIF (Cost Insurance and Freight).

Enquanto a região não se encontra na situação de normalidade, qualquer tipo de ação de ajuda gera custo. Quanto maior o tempo para atingir a situação de normalidade, maior o custo total do desastre. Foi utilizado custo de privação, definido como avaliações quantitativas do sofrimento Humano por falta de acesso de um bem ou serviço. Para exemplificar a definição deste custo, procurou-se responder a seguinte pergunta: quanto a falta de determinado produto custará por dia para a região atingida? A dificuldade para quantificar este custo, se deve a alta variação dos fatores, dos atributos considerados no atendimento aos desastres. Pode-se, por exemplo, utilizar um índice de vulnerabilidade da região para retratar este valor assim como, atribuir uma taxa que represente o valor de seguro para a região atingida ou, definir um valor adicional ao preço de forma a representar este custo de privação.

\subsection{Modelagem do problema}

O objetivo da metodologia proposta neste trabalho é atuar nas fases de preparação e resposta em uma operação humanitária. Atender as demandas das áreas afetadas no menor tempo possível, sob pena de vidas serem perdidas. Sendo assim, a modelagem foi flexível o bastante para considerar o 
tempo como variável e ponderar, negativamente, os possíveis atrasos. Com isso, a solução caminhou de modo a escolher fornecedores que entreguem os suprimentos o mais rápido possível, mesmo que, com maior custo. Pois, do ponto de vista de vidas em risco, a melhor opção não é, necessariamente, a de um fornecedor mais barato, mas daquele que conseguir atender a demanda na data solicitada.

Ou seja, o modelo deve conter o custo de privação, este valor foi adicionado ao preço de cada unidade do produto a cada dia em que o produto não se encontra no local específico. Na elaboração do problema, o custo de privação do produto por dia não atendido auxiliou a escolha do fornecedor no sentido de que, quando o fornecedor não tinha a quantidade solicitada para aquela data, mas para outra data posterior foi atribuído este custo de privação ao preço do fornecedor, representado no arco do fluxo associado a falta. Assim, o modelo definiu aqueles fornecedores com disponibilidade do produto, o mais próximo da data solicitada.

Foi proposto um modelo de grafo onde $T$ é o horizonte de tempo discretizado em períodos $1,2, \ldots, T ; K$ é o número de fornecedores; M é o número de localidades; $F_{k}$ é o vetor com capacidade do fornecedor $\mathrm{k}$ no horizonte de planejamento $\mathrm{T} ; F_{k}=\left(F_{1 k}, F_{2 k}, \cdots, F_{T k}\right) ; k=1, \cdots, K ; D_{m}$ é o vetor com a demanda da localidade $m$ no horizonte de planejamento T; $D_{m}=\left(D_{1 m}, D_{2 m}, \cdots, D_{T m}\right) ; m=$ $1, \cdots, M$. Têm-se dois tipos de nós: nó do tipo 1 é o par ordenado (capacidade do fornecedor, tempo) $=(F, t)$ e nó do tipo 2 é o par ordenado (demanda da localidade, tempo)= $(D, t)$. Têm-se três tipos de arcos que representam os fluxos: arco tipo 1 , fluxos associados ao estoque $\left(\left(F_{k}, t\right),\left(F_{k}, t+1\right)\right)$, arco tipo 2 , fluxos associados à falta $\left(\left(D_{m}, t\right),\left(D_{m}, t-1\right)\right)$ e arco tipo 3 , fluxos associados ao transporte $\left(\left(F_{k}, t\right),\left(D_{m}\right.\right.$, tempo $\left.\left.(k, m)\right)\right)$.

A Figura 1 apresenta o grafo desenvolvido, em que o rótulo do nó (capacidade do fornecedor, tempo) representa a quantidade de produto disponível à pronta entrega naquele período. O rótulo do nó (demanda da localidade, tempo) representa a quantidade necessária do produto no local do desastre no período solicitado. O objetivo é determinar quais fornecedores poderão atender ao evento de forma que o custo total de enviar o produto (suprimento) disponível através da rede seja minimizado, a fim de satisfazer a demanda dada. 
Figura 1

Modelo do grafo proposto

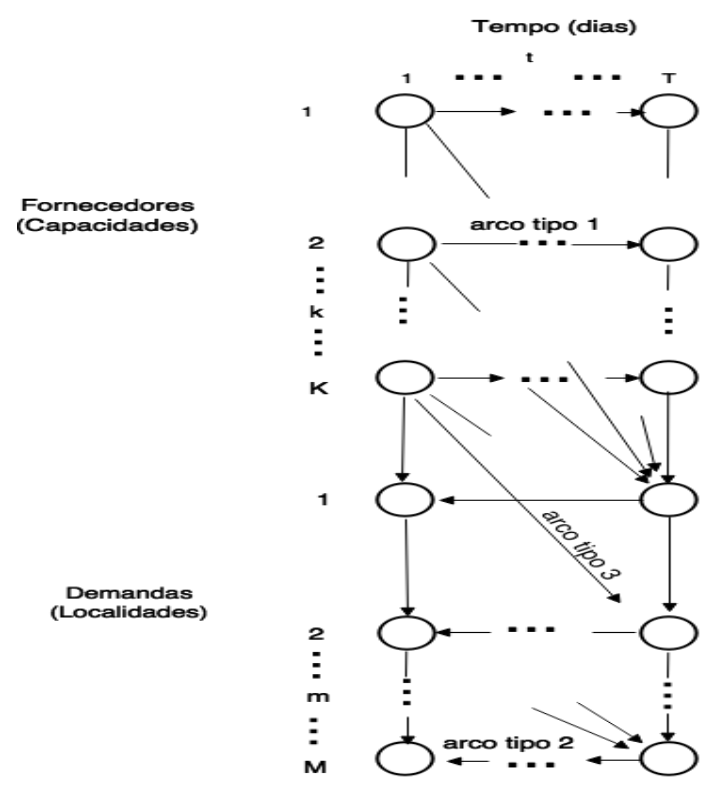

Fonte: Autores.

O método simplex especializado para resolver o problema de fluxo em redes, baseado na estrutura clássica de Kennington e Helgason (1980), foi então implementado.

$$
\text { Minimizar } \sum_{(i, j) \in A} c_{i j} x_{i j}
$$

s.a.

$$
\begin{array}{ll}
\sum_{(i, k) \in A} x_{i k}-\sum_{(k, j) \in A} x_{k j}=r_{k} & \forall k \in \mathrm{N} \\
0 \leq X_{i j} \leq u_{i j} & \text { para cada arco } \mathrm{i} \rightarrow j
\end{array}
$$

Buscou-se minimizar o custo total da provisão disponível através da rede a fim de satisfazer a demanda dada, sujeito à restrição de conservação de fluxo. Onde $c_{i j}$ é o custo unitário por fluxo através do arco $i \rightarrow j ; x_{i j}$ representa o fluxo através do arco $i \rightarrow j ; r_{k}$ define o fluxo líquido demandado no nó $\mathrm{k} ; u_{i j}$ é a capacidade dada no $\operatorname{arco} i \rightarrow j$. Considerou-se ainda que se $r_{k}>0$, o nó k é um nó de 
demanda com demanda igual a $r_{k}$; se $r_{k}<0$, o nó k é de oferta, com oferta igual a $\left|r_{k}\right|$ e, finalmente, se $r_{k}=0$, k é um ponto de transbordo. A figura 2 ilustra a modelagem proposta.

Importante lembrar que neste modelo, foi adicionado o custo de privação, para definir a escolha do fornecedor que irá atender a demanda mais próximo da data solicitada, ou seja, o método simplex irá definir por aquele que apresentar o menor custo mas, diferentemente da abordagem tradicional, este custo representa o custo total de aquisição do produto o qual é a soma do custo do produto com o custo de privação por dia, sendo que o custo do produto representa o custo efetivamente pago pela operação e o custo de privação, é o custo referente ao lead time, um valor virtual utilizado para auxiliar na escolha do fornecedor.

Figura 2

Modelagem proposta

INPUT

Fornecedores

Capacidade

Tempo

Demanda

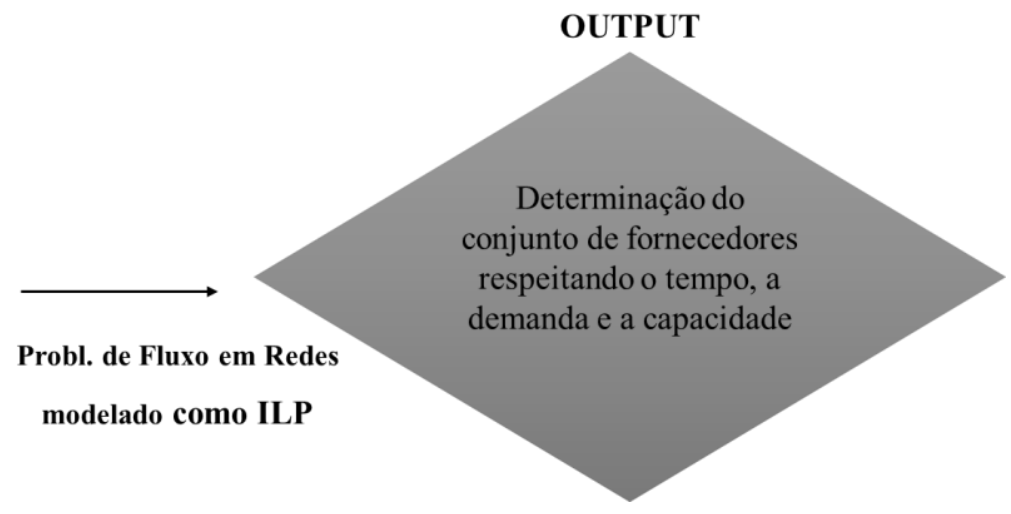

Fonte: Autores.

\section{Apresentação do cenário}

Santa Catarina é um dos 03 Estados do Brasil, localizado na região sul do país, com 295 municípios e 6.248.436 habitantes. Sua capital e sede do governo é a cidade de Florianópolis, localizada na Ilha de Santa Catarina (IBGE, 2010). Santa Catarina, dentre os estados brasileiros, destaca-se por ter um conjunto forte de elevações e depressões do terreno.

O estado de Santa Catarina é cenário de alagamentos, enchentes, inundações bruscas e graduais, escorregamentos, estiagens, vendavais, tornados, nevoeiros e ressacas. Segundo o Anuário brasileiro de desastres naturais (Ministério da Integração Nacional, 2014), o sistema climático na região está associado a eventos adversos, condições de estabilidade e pela ocorrência de fenômenos atípicos, como foi o caso do Furacão Catarina. 
Em outubro de 2014, temporais, fortes ventos e queda de granizo proveniente de um sistema de baixa pressão entre o Noroeste do RS e Litoral Sul de SC, acoplado a passagem de uma frente fria pelo oceano na altura do Litoral Sul de SC, favoreceu a forte instabilidade acompanhada de muitas descargas elétricas. Durante a tarde e noite da segunda-feira dia 13/10/2014, intensos núcleos de instabilidade provocaram fortes pancadas e chuva com raios em todas as regiões catarinenses. Os temporais vieram acompanhados de chuva forte localizada com volumes significativos.

No período de 13 a 25 de outubro de 2014, foram registradas queda de granizo e vendaval, em inúmeros municípios do Vale do Itajaí, Meio Oeste, Grande Florianópolis (incluindo a Capital) e, principalmente, na Serra Catarinense. A cidade de Lages, localizada na serra catarinense, e os municípios vizinhos foram muito afetados com a queda abundante de granizo e, até mesmo saraiva (gelo de formato irregular e com diâmetro bem maior do que o granizo), além de fortes rajadas de ventos.

Segundo o relatório de efeitos de impacto de evento adverso fornecido pela Secretaria de Defesa Civil de Santa Catarina, 105 mil pessoas foram afetadas, aproximadamente 8 mil desalojados, 159 desabrigados, 1óbito, 33.623 residências danificadas/destruídas e 132 instalações públicas foram danificadas.

Identificadas as reais dimensões dos danos e prejuízos provocados pelo evento, o Estado decretou situação de emergência, e solicitou recursos ao Governo Federal, através do plano detalhado de resposta. Segundo dados fornecidos pela Secretaria de Defesa Civil do estado recursos em caráter de assistência humanitária entorno de 3 milhões de reais foram disponibilizados do Fundo Estadual de Defesa Civil e Recursos do Governo Federal.

Segundo a Gerência de Logística e Mobilização da Secretaria de Defesa Civil de Santa Catarina (Defesa Civil, 2015) , diversos itens de assistência Humanitária foram solicitados como: colchões; kits de acomodação dormitório (colchão, cobertor, lençol, fronha e travesseiro); kits de limpeza; kits de higiene pessoal; telhas de $4 \mathrm{~mm}, 6 \mathrm{~mm}$ e $5 \mathrm{~mm}$; pares de cumeeira, pregos e lonas. Sendo que, a solicitação de telhas e lonas foi em grande quantidade devida o tipo de evento adverso.

Sendo assim, avaliaram-se as demandas destes produtos, por dia, para o atendimento emergencial nos municípios atingidos na região.

\subsection{Dados de entrada}

Segundo a Secretaria de Defesa Civil (SDC) de Santa Catarina, o produto emergencial mais utilizado foi a telha, para o atendimento emergencial nas regiões atingidas do estado. 
Considerando a emergência da situação foi primordial que o produto estivesse nas cidades atingidas o mais rápido possível. Buscou-se determinar quais fornecedores poderiam entregar o produto no tempo solicitado ou mais próximo disto considerando, ainda, o menor custo.

Sendo assim, avaliaram-se as demandas deste produto, por dia, para o atendimento emergencial nos municípios atingidos no estado. Esta abordagem considerou que no nível operacional, a organização local responsável ao atendimento emergencial, solicitou a atualização dos dados de cada fornecedor, analisou a oferta e verificou os dados de entrada dos fornecedores parceiros. Identificou quais fornecedores locais estão ativos e estão enquadrados nos critérios de qualidade solicitada.

Especificamente, para o desastre de Granizo, ocorrido em Santa Catarina, no ano de 2014, foi feita uma coleta de dados e análise junto a gerencia de logística e mobilização da SDC de Santa Catarina sobre os seguintes parâmetros de entrada (inputs):

a) Fornecedores: verificou-se o cadastro de fornecedores utilizados pela SDC para este tipo de produto e, devido à emergência e ao tipo de evento adverso, buscou-se uma relação de fornecedores em várias regiões e ainda, vários estados do Brasil. Considerou-se, arbitrariamente, para os fins da simulação, 300 fornecedores.

b) Capacidade: variou-se a capacidade dos fornecedores como capacidade total insuficiente e capacidade total suficiente. Para isto dividiu-se em três cenários, onde no primeiro a capacidade dos fornecedores estava variando de 1.000 a 30.000 unidades de telhas, no segundo variava de 1.000 a 10.000 unidades de telhas e no último de 1.000 a 90.000 unidades de telhas.

c) Data de solicitação dos pedidos: analisou-se os documentos referentes à solicitação dos pedidos do coordenador local de cada município atingido para o gerente de logística e mobilização e verificou-se em qual período de tempo, conforme o ciclo de vida de gestão dos desastres, ocorreu a maior demanda. Sendo assim, utilizou-se o intervalo de tempo do dia 13/10 até o dia 18/10. Ou seja, utilizou-se este intervalo para que, aleatoriamente, fosse relacionada à demanda por dia. Esta análise ajuda a determinar os padrões de fluxo do pedido, o que influencia no tempo de entrega.

d) Demanda: analisou-se a quantidade solicitada do produto por dia que cobriam o total dos dias de operação nos municípios atingidos. Esta análise ajudou a verificar que a quantidade solicitada variou entre 700 e 3.000 telhas por dia, sendo assim, trabalhou-se com quantidades variando aleatoriamente entre estes limites. 
e) Preço: verificou-se o preço atribuído por cada fornecedor para o produto. Definiu-se então, um intervalo onde o preço mínimo é de $\mathrm{R} \$ 7,00$ e o preço máximo é de $\mathrm{R} \$ 12,00$ por telha.

f) Custo de Privação do produto: devido à urgência de necessidade do produto na região, arbitrou-se a fim de experimentação do modelo, juntamente com especialistas da SDC, que a falta por dia do produto neste evento adverso deveria ser maior que o limite superior do preço do fornecedor. Sendo assim, verificou-se que o valor de $\mathrm{R} \$ 20,00$ por unidade de telha serviria como um bom indicador para a simulação.

A gravidade do evento impossibilitou que fornecedores locais estivessem disponíveis para atender a demanda solicitada, por isso, considerou-se fornecedores de outros estados brasileiros.

\subsection{Implementação computacional}

O método simplex especializado para resolver o problema de fluxo em redes foi implementado utilizando a linguagem de programação Java. Utilizou-se o simulador Anylogic ${ }^{\circledR}$, para representar os resultados da modelagem proposta, o qual possibilitou a visualização na linha do tempo para o cenário analisado. Considerou-se para a simulação do cenário o período ocorrido no início do atendimento ao desastre, dia 13 de outubro até o dia 29 de outubro, quando a organização humanitária iniciou o processo de desmontagem da estrutura de atendimento emergencial.

O algoritmo do problema de fluxo em redes roda a cada dia com um horizonte de tempo de 7 dias, ou seja, considerou-se sempre os fornecedores disponíveis nos próximos 7 dias.

\section{Análise dos resultados obtidos}

A Figura 3 apresenta uma forma geral do resultado do problema de fluxo em redes do cenário analisado. Cada um dos Itens A, B, C D e E serão explicitados, individualmente, a seguir. 


\section{Resultado geral}

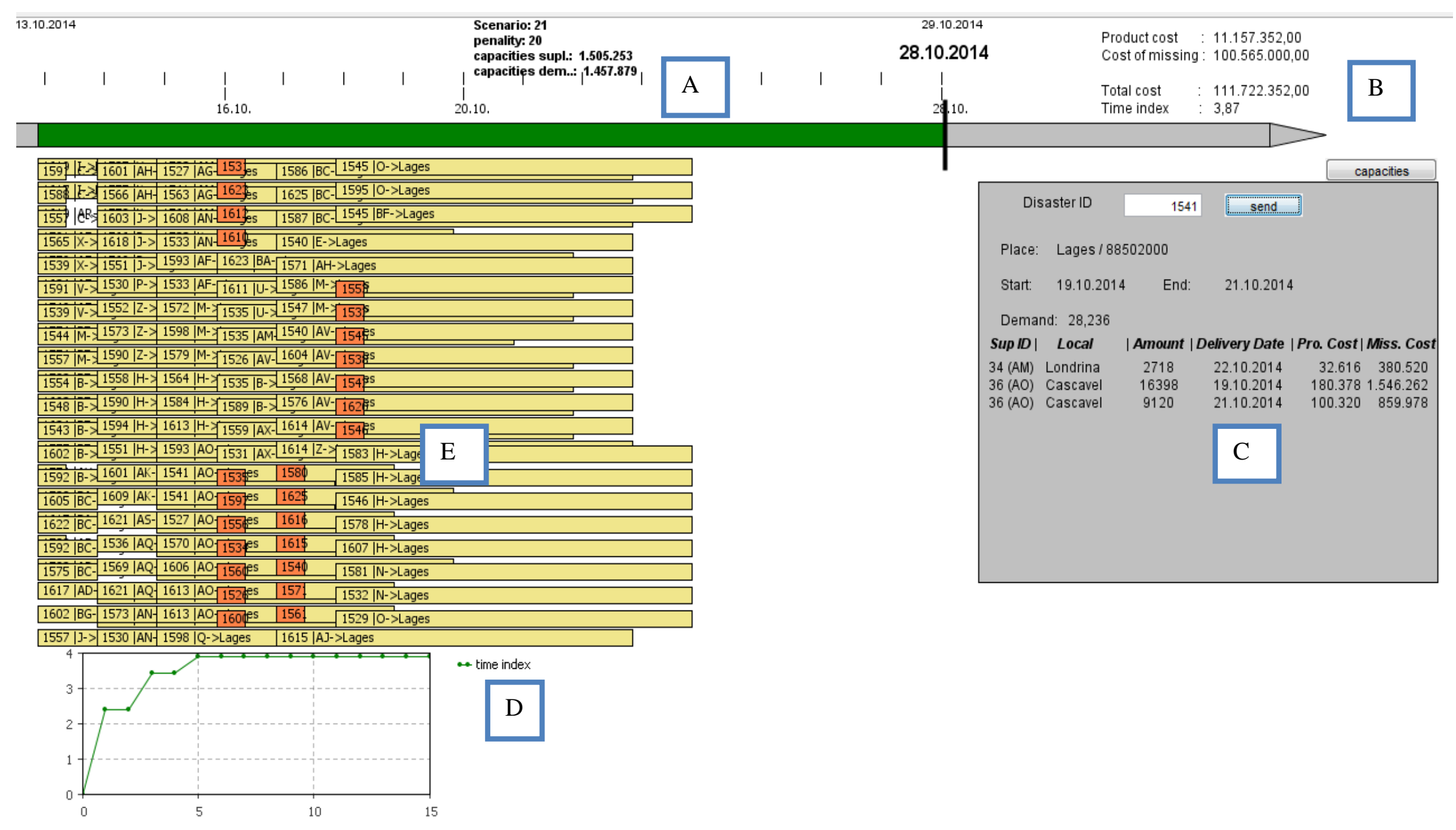

Fonte: Autores. 
A: Régua do Tempo - Indica o período, data de início e fim em que é feita a operação total. Esta régua auxilia na visualização do período em que a operação de atendimento ocorreu.

B: Valores dos Custos e Índice de Desempenho - Indica o custo da operação no período. Apresenta o custo total de aquisição do produto (Total cost) que é a soma do custo do produto (Product cost) e o custo de privação por dia (Cost of missing).

C: Quadro dos Resultados de Atendimento Específico por Local de Demanda - Este quadro representa como foi distribuído o atendimento da demanda no local solicitado. Indica o local da demanda, a data inicial e a data final em que a demanda foi atendida e a quantidade total demandada. Apresenta qual fornecedor atendeu àquela demanda, quanto do produto solicitado em qual dia foi atendido e indica, também, o custo da operação por dia atendido. Este custo é representado separadamente pelo custo do produto (Pro. cost) e pelo custo de privação (Miss. Cost), sendo que o custo do produto representa o custo efetivamente pago pela operação e o custo de privação, é o custo referente ao lead time, um valor virtual utilizado para auxiliar na escolha do fornecedor.

D : Gráfico do índice do Tempo de Atendimento- Este gráfico representa o comportamento do índice do tempo (número de dias para o atendimento à demanda) .Quanto menor o tempo para atender totalmente a demanda melhor o desempenho do sistema.

E: Régua do Tempo de atendimento - Apresenta o espaço onde se pode visualizar o tempo de atendimento de cada demanda. É representado pelo Id da demanda, nome do fornecedor e local da demanda. Por exemplo, a demanda Id 1607 foi atendida pelo fornecedor $\mathrm{H}$, e o local da demanda é a cidade de Lages. As demandas que não foram atendidas estão representadas pelas réguas escuras (em vermelho).

A Figura 4 exibe uma demanda que não foi totalmente atendida e a Figura 5 exibe uma demanda que foi atendida.

\section{Figura 4}

\section{Demanda não atendida}

\begin{tabular}{|c|c|c|c|c|c|}
\hline \multicolumn{2}{|c|}{ Disaster ID } & 1382 & send & & \\
\hline \multicolumn{6}{|c|}{ Place: Lages/88502000 } \\
\hline Start: & 18.10 .2014 & End: & 25.10 .2014 & & \\
\hline \multicolumn{6}{|c|}{ Demand: 23,973} \\
\hline Sup ID | & Local & | Amount | & Delivery Date | & Pro. Cost | & Miss. Cos1 \\
\hline $\begin{array}{c}35 \text { (AN) } \\
6(\mathrm{~F})\end{array}$ & $\begin{array}{l}\text { Porto Alegre } \\
\text { Xanxere }\end{array}$ & $\begin{array}{l}6939 \\
5304\end{array}$ & $\begin{array}{l}25.10 .2014 \\
25.10 .2014\end{array}$ & $\begin{array}{l}69.390 \\
53.040\end{array}$ & $\begin{array}{l}971.460 \\
742.560\end{array}$ \\
\hline 39 (AR) & Aguas Frias & 4732 & 25.10 .2014 & 47.320 & 662.480 \\
\hline${ }^{\star}$ F ault* & - & 6998 & 25.10 .2014 & 0 & 0 \\
\hline
\end{tabular}

Fonte: Autores.
Figura 5

\section{Demanda atendida}

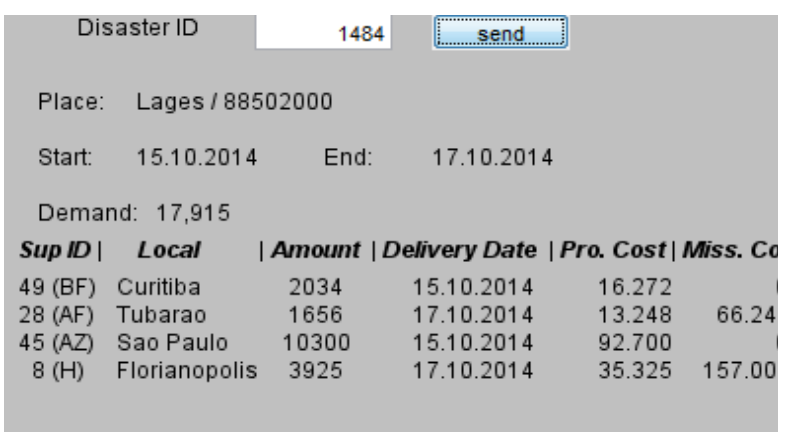

Fonte: Autores 
Na Figura 4 lê-se que foi solicitado no dia 18/10 uma quantidade 23.973 telhas para a cidade de Lages, localizada na serra catarinense. Uma parte desta demanda foi atendida pelos fornecedores parceiros localizados em Porto Alegre, Xanxerê e Águas Frias, mas, a capacidade dos fornecedores parceiros não foi suficiente para atender a demanda solicitada no prazo de sete dias, ou seja, faltaram 6.998 telhas para atender a região de Lages no período solicitado.

Na Figura 5, foi solicitado no dia 15/10 uma quantidade de 17.915 telhas, também para a cidade de Lages. Fornecedores parceiros localizados em Curitiba, Tubarão, São Paulo e Florianópolis atenderam a demanda solicitada em menos de 2 dias sendo que os fornecedores de Curitiba e São Paulo conseguiram atender a demanda no mesmo dia em que foi solicitada.

As necessidades de cada município foram atendidas conforme a disponibilidade informada por cada fornecedor parceiro e, devido ao custo de privação, o programa atendeu no tempo mais próximo da data de demanda. Quanto menor o tempo para atender totalmente a demanda melhor o desempenho do sistema. Para medir o desempenho foi introduzido o índice de tempo (time index). Este índice é calculado utilizando a média do atendimento em dias para cada demanda ao longo do tempo do cenário.

\section{Considerações finais}

Desastres exigem respostas imediatas, ou seja, suprimentos certos, na hora certa, no lugar certo e distribuídos nos locais e pessoas certas. Requer a implementação de estratégias, que deve iniciar na fase de pré-desastres, ou seja, antes do desastre ocorrer. Ações voltadas a fase de resposta passam a ser direcionadas por planejamentos considerados no nível tático e operacional, tendo a logística como atividade central.

As ações logísticas são propostas com o objetivo de auxiliar as diferentes atividades referentes as operações de resposta a desastre (processos aduaneiros, transporte, armazenagem, aquisição, distribuição, relacionamento com fornecedores, entre outros), as quais são propostas levando em consideração as atividades mapeadas no modelo e planejadas com antecedência.

Desta forma, a tomada de decisão referente a gestão dos recursos, foi trabalhada como um modelo de apoio as operações humanitárias em um evento de desastre natural ocorrido na serra catarinense, com destaque para a função de aquisição de suprimentos durante a fase de resposta. Com a estrutura de demanda, determinados fornecedores foram selecionados com a possibilidade de entregar os itens em curto prazo, utilizando um modelo de fluxo em redes adaptado e considerando, a inclusão do custo de privação. 
No resultado do cenário estudado, a condição de o produto estar o mais rápido possível no local de demanda foi satisfeita e foi utilizada a capacidade dos fornecedores nas datas mais próximas aos eventos conforme apresentado no objetivo do modelo.

A adaptação do problema do fluxo em redes para o contexto da LH é uma das principais contribuições do trabalho, pois, diferentemente da Logística Tradicional, em que o resultado do método simplex no fluxo em redes prioriza o menor custo, na Logística Humanitária, devido a inclusão do custo de privação, o programa prioriza o tempo mais próximo da data de demanda, utilizando para isto, o fornecedor com capacidade suficiente para atender na data solicitada ou o mais próximo possível desta data. Quanto menor o tempo para atender totalmente a demanda melhor o desempenho do sistema.

Este modelo pode vir a auxiliar os tomadores de decisão em organizações humanitárias quanto a necessidade de agilidade na aquisição de suprimentos por via de fornecedores tanto internos quanto externos, na formalização das relações e processos de compra de produtos e na contratação de serviços.

O planejamento de métodos de compras com fornecedores, a consolidação de solicitações de produtos, a definição, preparação e aferição da lista de itens básicos de produtos a serem adquiridos em eventos de desastres, e o desenvolvimento e/ou atualização da lista de fornecedores, são atividades que requerem total atenção dos tomadores de decisão nas organizações humanitárias quer seja no nível nacional quanto internacional.

O estudo proposto limitou-se ao desenvolvimento de um modelo matemático e computacional para a função de aquisição.

Para futuros estudos, novas variáveis podem ser introduzidas tais como a modelagem da escolha do caminho mais curto ou tipos de modais apropriados. Assim como desenvolver formas para o cálculo do custo de privação, considerando as especificidades da LH para os diferentes tipos de desastres e incluir este custo de privação como um índice na estrutura clássica do método simplex de Kennington e Helgason (1980).

Desta forma, para qualquer tipo de desastres, dentro das condições estabelecidas, a escolha do fornecedor e a distribuição dos itens emergenciais solicitados poderá será atendida no tempo certo e assim, auxiliar no atendimento a um maior número de pessoas atingidas.

\section{Agradecimentos}

Os autores agradecem aos especialistas da SDC/SC.

\section{Referências}

Balcik, B., Beamon, B. M., Krejci, C, Muramatsu, K. M., \& Ramirez, M. (2010). Coordination in humanitarian relief chains: Practices, challenges and opportunities. International Journal 
Production Economics. Science Direct, 12(6), 22-34.

https://doi.org/10.1016/j.ijpe.2009.09.008

Balcik, B., \& Beamon, B. M. (2008). Facility location in humanitarian relief. International Journal of Logistics: Research and Applications, 11(2), 101-121. https://doi.org/10.1080/13675560701561789

Blecken, A. (2010). Supply chain process modelling for humanitarian organizations. Journal of Physical Distribution \& Logistics Management, 40(8/9), 675-692. https://doi.org/10.1108/09600031011079328

Cauchick M. P. (2010). Metodologia de Pesquisa em Engenharia de Produção e Gestão de Operações. Elsevier, Rio de Janeiro. ISBN (versão digital): 978-85-352-9135-3.

Cobrade (2020). Classificação e codificação brasileira de desastres. Disponível em: https://www.bombeiros.go.gov.br/wp-content/uploads/2012/06/1.Codifica\%C3\%A7\%C3\%A3o-e-Classifica\%C3\%A7\%C3\%A3o-Brasileira-de-DesastresCOBRADE2.pdf

Coughlan, P., \& Coghlan, D. (2002) Action research for operations management. International Journal of Operations \& Production Management, 22(2), 220240. https://doi.org/10.1108/01443570210417515

Defesa Civil. (2015). Defesa Civil - Santa Catarina. Disponível em https://www.defesacivil.sc.gov.br/

Ertem, M. A., \& Buyurga, N. N. (2011) An auction-based framework for resource allocation indisaster relief. Journal of Humanitarian Logistics and Supply Chain Management, 1(2), 170-188. https://doi.org/10.1108/20426741111158412

Falasca, M., \& Zobel, C. (2011). A two-stage procurement model for humanitarian relief supply chains, Journal of Humanitarian Logistics and Supply Chain Management, 1(2), 151-169 https://doi.org/10.1108/20426741111188329

IBGE. (2010). Instituto Brasileiro de Geografia e Estatística. Disponível em: https://www.ibge.gov.br/. Acesso em 23 mar 2015.

Kennington, J. L., \& Helgason, R.V. (1980). Algorithm for Network Programming. New York:, John Willey \& Sons. https://doi.org/10.1002/net.3230120107

Lima, F. S., Oliveira, D., \& Gonçalves, M. B. (2014). Formação de clusters para o gerenciamento da cadeia de suprimentos em operações humanitárias. Exacta - EP, 12(1), 55-68. https://doi.org/10.5585/exactaep.v12n1.4696

Lima, F. S., Eyerkaufer, M. L., Gonçalves, M. B. (2017). Capítulo 5 - Gestão de Desastres. In: Adriana Leiras; Hugo Tsugunobu Yoshida Yoshizaki; Márcia Marcondes Altimari Samed;Mirian Buss Gonçalves. (Org.). Logistica Humanitária. 1ed. Rio de Janeiro: Elsevier, 57-77. ISBN 978-85-3528795-0

López-Vargas, J. C., Cárdenas-Aguirre, D. M. (2018). Factores de influencia en la coordinación logística para la preparación y atención de desastres - Una revisión de literatura. Revista EIA, 15(30), 41-56. https://doi.org/10.24050/reia.v15i30.1146 
Marco de Sendai. (2015). Marco de Sendai para a Redução do Risco de Desastres 2015- 2030.

Disponível em:

https://www.mdr.gov.br/images/stories/ArquivosDefesaCivil/ArquivosPDF/Sendai_Framework _for_Disaster_Risk_Reduction_2015-2030-Portugus.pdf

Ministério da Integração Nacional. (2014). Anuário brasileiro de desastres naturais. Ministério da Integração Nacional. Secretaria Nacional de Proteção e Defesa Civil. Centro Nacional de Gerenciamento de Riscos e Desastres. - Brasília: CENAD. Disponível em:

https://www.mdr.gov.br/images/stories/ArquivosDefesaCivil/ArquivosPDF/publicacoes/Anurio -Brasileiro-de-Desastres-Naturais-2013.pdf

Olsen, G., Carstensen, N. \& Hoyen, K. (2003). Humanitarian crisis: What determines the level of emergency assistance. Media coverage, donor interest and the AID business. Disasters, 27(2), 109-26. https://doi.org/10.1111/1467-7717.00223

ONU. (2009). Organização das Nações Unidas. Disponível em: https://nacoesunidas.org/

Rodriguez, J., Vitoriano, B., Montero, J. (2012). A general methodology for data-based rule building and its application to natural disaster management. Computers \& Operations Research. https://doi.org/10.1016/j.cor.2009.11.014

Samed, M. M. A., Gonçalves, M. B. (2017). Capítulo 3 - Introdução à Logística Humanitária In: Adriana Leiras; Hugo Tsugunobu Yoshida Yoshizaki; Márcia Marcondes Altimari Samed;Mirian Buss Gonçalves. (Org.). Logistica Humanitária. 1ed.Rio de Janeiro: Elsevier, 27-37. ISBN 978-85-3528795-0

Schultz, S. F. (2008). Disaster Relief Logistics: Benefits of and Impediments to Horizontal Cooperation between Humanitarian Organizations. Doctoral thesis, Technischen Universität Berlin, Germany. http://dx.doi.org/10.14279/depositonce-2086

Schulz, S. F., \& Heigh, I. (2009). Logistics performance management in action within a humanitarian organization, Management Research News, 32(11). https://doi.org/10.1108/01409170910998273

Tatham, P. \& Pettit, S. (2010). Transforming humanitarian logistics: the journey to supply network management. International Journal of Physical Distribution \& Logistics Management, 40(8/9), 609-622. https://doi.org/10.1108/09600031011079283

Taupiac, C. (2001). Humanitarian and development procurement: a vast and growing market, International Trade Forum, 4, 7-10. https://www.tradeforum.org/Humanitarian-andDevelopment-Procurement---A-Vast-and-Growing-Market/

Thomas, A. (2004). Elevating Humanitarian Logistics. International Aid \& Trade Review, 102-106

Trestrail, J., Paul, J., \& Maloni, M. (2009), Improving bid pricing for humanitarian logistics, International Journal of Physical Distribution \& Logistics Management, 39(5), 428-41. https://doi.org/10.1108/09600030910973751

Trunick, P. (2005). Special report: delivering relief to tsunami victims. Logistics Today, 46(2), 1-3.

Van Wassenhove, L. V. (2006). Humanitarian Aid Logistics: Supply Chain Managent in High Gear, Journal of the Operational Research Society, 57(5), 475-489.

https://doi.org/10.1057/palgrave.jors.2602125 